\title{
Incidencia del uso de gasolina extra y mezcla al $5 \%$ con etanol anhidro en los microfiltros de los inyectores multipunto
}

\section{Incidence of the use of extra gasoline and mixing to the $5 \%$ with anhydrous ethanol in the microfilters of the multipoint injectors}

\author{
Juan Rocha-Hoyos ${ }^{1}$, Edilberto Llanes-Cedeño ${ }^{1}$, Jefferson Andrade-Villarreal,3, \\ Luis Caiza-Quishpe ${ }^{1,2,3}$, Julio Leguísamo-Milla ${ }^{1}$
}

\begin{abstract}
Resumen
El objetivo de este estudio fue analizar los depósitos en inyectores con una mezcla de combustible de 87 octanos con porcentajes de etanol anhidro al 5 \% y gasolina de 87 octanos. La fase experimental se llevó a cabo en dos vehículos con motor de encendido a una altura de prueba de 2810 m.s.n.m. al recorrer, cada uno, una distancia de 3000 km dentro del Distrito Metropolitano de Quito. Se aplicaron dos métodos: Estereoscopía o Visión para el análisis de los microfiltros de los inyectores; y el análisis químico semicuantitativo Microscopía Electrónica de Barrido para las muestras de los residuos originados en los filtros. En el caso de la estereoscopia, en ambos vehículos, se produjo una reducción de la obstrucción de los filtros cuando se usa gasolina con etanol anhidro al 5 \%. La microscopía electrónica de barrido permitió concluir que hay una mayor presencia del elemento azufre con un $4 \%$ en los residuos cuando se usa gasolina con un $5 \%$ de etanol, a diferencia del $1 \%$ en los residuos cuando se aplica gasolina de 87 octanos.
\end{abstract}

\section{Palabras clave}

Microfiltro; contaminantes; etanol; gasolina; inyectores.

\begin{abstract}
The purpose of the study was to analyze the deposits in injectors with fuel mixture of 87 octane with percentages of anhydrous ethanol at $5 \%$, and 87 octane gasoline. The experimental phase was carried out in two ignition engine vehicles at a test height of $2810 \mathrm{msnm}$ when traveling, each one, a distance of $3000 \mathrm{~km}$ inside the Metropolitan District of Quito. Two methods were applied: Stereoscopy or Vision for the analysis of the microfilters of the injectors, and the semiquantitative chemical analysis Scanning Electron Microscopy for the samples of the residues originated in the filters. In the case of Stereoscopy, in both vehicles, a reduction of clogging of the filters occurred when using gasoline with $5 \%$ anhydrous ethanol. The Scanning Electron Microscopy allowed to conclude that there is a greater presence of the sulfur element with $4 \%$ in the residues when using gasoline with $5 \%$ ethanol, unlike $1 \%$ in the waste when 87 octane gasoline is applied.
\end{abstract}

\section{Keywords}

Microfilter; pollutants; ethanol; gasoline; injectors.

\section{Introducción}

En 2017 los vehículos vendidos en el Ecuador ascendieron a 105101 unidades; la participación porcentual en venta de vehículos por el tipo de combustible fue: 77.6 \% vehículos a gasolina, 19.2 \% vehículos a diésel, 3.1 \% vehículos híbridos y $0.1 \%$ vehículos eléctricos (CINAE, 2018). El Emisiones, (antonio.llanes@uisek.edu.ec, carlos.rocha@uisek.edu.ec, julio.leguisamo@uisek.edu.ec, lcaiza.mdm@uisek. edu.ec). 
gran crecimiento del parque automotor en el Distrito Metropolitano de Quito (DMQ), cuya tasa de incremento supera el 7.4 \% anual, alcanzando los 438815 vehículos en el 2016 (Secretaría de Ambiente, 2016).

Según Páez (2008), la altitud de Quito a 2800 m.s.n.m., en promedio, es una razón para que el aire de Quito tenga naturalmente menos oxígeno, lo cual conspira contra la eficiencia de la combustión, provocando que los equipos que combustionan combustibles fósiles, como los generadores, incineradores industriales y los motores de los vehículos, consuman mayor cantidad de combustible y, paralelamente, generen mayor cantidad de contaminantes en comparación con proceso similares que se realizan a nivel del mar (Rocha-Hoyos, Tipanluisa, Zambrano, y Portilla, 2018; Merchán, Moromenacho, Milla, y Cedeño, 2018). El comportamiento adecuado del sistema de inyección de un motor de combustión interna de encendido provocado (MEP) depende de la cantidad y calidad del aire y combustible para llegar a una combustión completa. El diseño y estado del inyector influye directamente en la inyección de la cantidad del combustible al motor para llegar a una conveniente combustión sin fugas ni escapes de carburante (Llanes, Rocha-Hoyos, Peralta, y Leguísamo, 2018). En su estudio Peng (2017) compara el efecto de la gasolina pura sin plomo y la gasolina sin plomo mezclada con biocombustibles en el desgaste del inyector de gasolina y las emisiones de escape; se agregan biocombustibles con una concentración de 0, 1, 2, 5 y 10 \% a la gasolina sin plomo para formar combustibles mezclados con etanol, después de 4000 horas de funcionamiento del motor a 500 rpm, los resultados del experimento mostraron que en comparación con la gasolina pura sin plomo, el uso de combustibles mezclados con etanol en los motores de gasolina de cuatro tiempos generaban menos emisiones de escape de monóxido de carbono (CO), hidrocarburos $(\mathrm{HC})$ y óxido de nitrógeno (NOx); y que la rugosidad superficial del émbolo del inyector disminuye de 0.378 a $0.337 \mu \mathrm{m}$. El-Faroug, Yan, Luo, y Fiifi-Turkson (2016) indican que el uso de combustible con etanol muestra presiones de trabajo mayores, y una temperatura inferior en el múltiple de admisión, las cuales son obtenidas con el uso de mezclas de etanol hidratado; como resultado obtuvieron una mayor eficiencia volumétrica.

Los depósitos en el inyector de los motores de encendido directo a gasolina (conocido como inyectores $\mathrm{GDI}$ ) son un fenómeno común y afectan en gran medida el comportamiento de la pulverización y, en consecuencia, el rendimiento de la combustión y las emisiones (Arters, y Macduff, 2000; Llanes-Cedeño, Carguachi-Caizatoa, y Rocha-Hoyos, 2018). Por su parte, Wang et. al. (2017) realizaron un análisis numérico del efecto del depósito en el flujo de la boquilla y las características de pulverización de los inyectores GDI; los resultados de las simulaciones de flujo de la boquilla resaltan que la superficie rugosa de los depósitos conduce a un inicio de cavitación adicional y restringe el área de flujo, causando pérdidas en el flujo de masa. Los depósitos en el interior del inyector restringen la recirculación del aire, las simulaciones de pulverización han demostrado que los depósitos conducirían a una mayor penetración de pulverización, un ángulo de cono de pulverización más pequeño y diámetros de gotas más grandes. Una cantidad relativamente pequeña de depósitos en el inyector restringe el flujo de combustible, distorsionan los patrones de rociado y afectan la combustión dentro del cilindro. Esto es motivo del aumento del consumo de combustible, mayores emisiones e incluso provoca problemas de conducción del vehículo, tales como un fallo de encendido (Zhao, Lai, y Harrington, 1999; Aradi, Evans, Miller, y Hotchkiss, 2003).

Song, Xiao, Chen, y Huang (2016) estudiaron el efecto del depósito en el comportamiento de rociado de los inyectores GDI de varios orificios con un inyector de coque que se utilizó 
en un vehículo durante 58000 km y afirmaron que el depósito aumenta el ángulo del cono de rociado y reduce la penetración. Lindgren, Skogsberg, Sandquist, y Denbratt (2003) estudiaron el efecto del depósito en inyectores concluyendo que el inyector sucio producía un pre-jet más denso y más rápido en comparación con el inyector limpio. Por lo expuesto, el presente estudió tuvo como objetivo determinar la influencia del uso de gasolina extra y gasolina extra con $5 \%$ de etanol anhidro en los inyectores de motor a gasolina mediante el análisis de los depósitos formados en los microfiltros para la evaluación de su uso.

\section{Materiales y métodos}

En la presente investigación se adoptó una estrategia para que el procedimiento heurístico permita la toma de decisiones en condiciones específicas, además, las tácticas son siempre conscientes e intencionales y están dirigidas a un objetivo relacionado con el estudio. Una técnica de investigación es un procedimiento algorítmico (Latorre y Seco, 2013; Vega, Llanes, Molina, Rocha-Hoyos, 2018), que dará forma a la realización de la investigación planteada dividida en tres partes con pasos lógicos y ordenados a seguir para concretar el estudio (Guardia, Márquez, Sánchez, Llanes-Cedeño, Rocha-Hoyos y Peralta, 2018; Llanes-Cedeño, Rocha-Hoyos, Peralta, Martínez, y Celi, 2018). Al respecto de su alcance es del tipo experimental, pues evalúa las variables de forma controlada.

\subsection{Selección de vehículos y combustibles}

La selección del vehículo se realizó con base en las características del parque automotor del país, considerando que el DMQ es el más representativo en automotores. Se prefieren dos vehículos de marca Chevrolet de distinto cilindraje, por ser la marca más vendida por varios años en el país, que ha llegado a una participación del 34.8 \% en ventas en el año 2017 (CINAE, 2018). Se seleccionan los automóviles Chevrolet Aveo 1500 cc año 2010, debido a que es el modelo más vendido en la actualidad, teniendo una participación del $14.5 \%$ en ventas en el parque automotor ecuatoriano; y el automóvil modelo Chevrolet Corsa 1800 cc año 2005 considerando que la edad promedio del parque automotor circulante en el país es de 13 años (CINAE, 2018; Andrade, 2018). En la Tabla 1 se detallan las especificaciones de los vehículos seleccionados. Las propiedades fisicoquímicas de los combustibles utilizados se presentan en la Tabla 2.

Tabla 1. Especificaciones técnicas de los vehículos seleccionados

\begin{tabular}{|l|l|l|}
\hline \multicolumn{1}{|c|}{ Especificación } & \multicolumn{1}{|c|}{$\begin{array}{c}\text { Vehículo 1 } \\
\text { (Chevrolet Aveo Family) }\end{array}$} & \multicolumn{1}{c|}{\begin{tabular}{c}
\multicolumn{1}{c|}{ Vehículo 2 } \\
(Chevrolet Corsa Evolution)
\end{tabular}} \\
\hline Año & 2010 & 2005 \\
\hline Kilometraje & 455733 & 188900 \\
\hline Posición de motor & Delantera transversal & Delantera transversal \\
\hline No. de cilindros & 4 en línea & 4 en línea \\
\hline Transmisión & Manual & Manual \\
\hline Cilindraje (cm $\left.{ }^{3}\right)$ & 1500 & 1800 \\
\hline Potencia (Hparpm) & $83 @ 5600$ & $107 @ 5400$ \\
\hline Torque (Nm@rpm) & $127 @ 300$ & $163 @ 2800$ \\
\hline Relación de compresión & $9: 05: 01$ & $9: 04: 01$ \\
\hline
\end{tabular}


Tabla 2. Propiedades de los combustibles (Llanes et al., 2018)

\begin{tabular}{|c|c|c|c|c|}
\hline $\begin{array}{l}\text { Propiedades del } \\
\text { combustible }\end{array}$ & Normas & $\begin{array}{c}\text { Norma } \\
\text { NTE INEN: } \\
935: 2012\end{array}$ & $\begin{array}{c}\text { Gasolina } \\
87 \text { octanos }\end{array}$ & $\begin{array}{c}\text { Gasolina } 87 \\
\text { octanos con } 5 \% \\
\text { de etanol }\end{array}$ \\
\hline Números de octanos & ASTM D2699 & 87 min. & 88.3 & 85.1 \\
\hline \multicolumn{5}{|l|}{ Curva de Destilación } \\
\hline T10-10\% evap., ${ }^{\circ} \mathrm{C}$ & \multirow{4}{*}{ ASTM D88 } & 70 max. & 50 & 56 \\
\hline T50-50\% evap., ${ }^{\circ} \mathrm{C}$ & & $77-121$ & 99 & 105 \\
\hline T90-90\% evap., ${ }^{\circ} \mathrm{C}$ & & 189 máx. & 163 & 167.5 \\
\hline Residuo \% & & 98 min. & 98.5 & 98.5 \\
\hline Presión de vapor de Reid, kPa & ASTM D4953 & 60 máx. & 58.6 & 50 \\
\hline $\begin{array}{l}\text { Corrosión a la lámina de cobre } \\
\left(3 \text { h a } 50^{\circ} \mathrm{C}\right)\end{array}$ & ASTM D130 & 1 & 1 & 1 \\
\hline Contenido de azufre & ASTM D7220 & 650 máx. & 169 & 190 \\
\hline Contenido de gomas $\left(\mathrm{mg} / \mathrm{cm}^{3}\right)$ & ASTM D381 & 3 máx. & 1 & 1 \\
\hline
\end{tabular}

\subsection{Caracterización de las muestras por estereoscopia}

La estereoscopía o visión se realiza a los microfiltros de los inyectores de los vehículos seleccionados para obtener una vista tridimensional más detallada de los mismos. Los microfiltros de los inyectores de los vehículos, modelos Aveo y Corsa, se analizan al recorrer una distancia de 3000 km dentro del DMQ y con diferentes combustibles: gasolina de 87 octanos y gasolina 87 de octanos con $5 \%$ de etanol anhidro. Para la visión estereoscópica de las muestras se utiliza el estereoscopio OLYMPUS SZX2-ILLD (Figura 1), y en la Tabla 3 se muestran las especificaciones técnicas. El procedimiento de este ensayo se realizó bajo la norma ASTM E381-17 (Standard Method of Macrotech Testing Steel Bars, Billets, Blooms and Forgins) (Proaño, 2018).

Tabla 3. Especificaciones del equipo OLYMPUS SZX2-ILLD (OLYMPUS, 2018)

\begin{tabular}{|c|c|c|c|}
\hline Características & Especificación & & Detalles \\
\hline \multirow{4}{*}{ Método de observación } & \multicolumn{2}{|c|}{ Fluorescencia (excitaciones verde/azul) } & $\sqrt{ }$ \\
\hline & \multicolumn{2}{|c|}{ Fluorescencia (excitaciones ultravioleta) } & $\sqrt{ }$ \\
\hline & Campo claro & & $\sqrt{ }$ \\
\hline & Campo oscuro & & $\sqrt{ }$ \\
\hline \multirow{2}{*}{ Zoom } & Motorizado & & $\sqrt{ }$ \\
\hline & AS & & Incorporado \\
\hline Revólver porta objetivos & Manual & Tipo estándar & $\sqrt{ }$ \\
\hline \multirow[t]{3}{*}{ Tubos de observación } & $\begin{array}{l}\text { Gran campo (número de cam- } \\
\text { po 22) }\end{array}$ & Trinocular & $\sqrt{ }$ \\
\hline & Ángulo de inclinación del tubo & & $\begin{array}{l}5-45^{\circ} \\
30^{\circ}\end{array}$ \\
\hline & \multicolumn{2}{|c|}{ Selección de la trayectoria de luz del cabezal trinocular } & $\begin{array}{l}50 \%: 50 \%, 0 \%: 100 \% \\
0 \%: 100 \%, 100 \%: 0 \%\end{array}$ \\
\hline
\end{tabular}


Figura 1. Estereoscopio OLYMPUS SZX2-ILLD

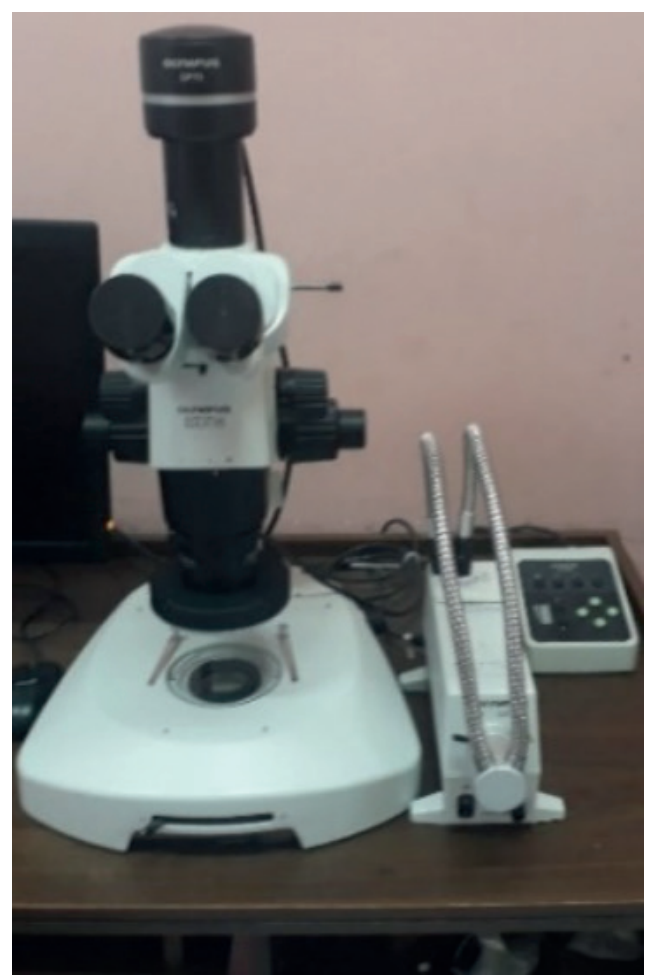

\subsection{Caracterización de las muestras por análisis de Microscopia Electrónica de Barri- do (MEB)}

Se realizó un análisis químico semicuantitativo por microscopía electrónica de barrido con microanálisis de Rayos $X$ a la granulométrica de los depósitos del inyector; a partir de los depósitos sin tratamiento previo, los que se extraen de los microfiltros de los inyectores de los vehículos seleccionados (Pérez, Moya, y Coello, et al., 2018; Villavicencio Cevallos, 2016). Los residuos de los microfiltros de los inyectores de los vehículos se obtienen al recorrer una distancia de 3000 $\mathrm{km}$, dentro del DMQ y con dos diferentes combustibles: gasolina extra y gasolina extra con $5 \%$ de etanol anhidro. En la Figura 2 se muestra el equipo utilizado y en la Tabla 4 se muestran las especificaciones técnicas del mismo.

Tabla 4. Especificaciones técnicas del microscopio electrónico Tescan-Vega LMU (Tescan, 2018)

\begin{tabular}{|l|l|}
\hline \multicolumn{1}{|c|}{ Parámetro } & \multicolumn{1}{c|}{ Especificación } \\
\hline Pistola de electrones & Cátodo calentado de tungsteno \\
\hline Resolución & $3 \mathrm{~nm}$ a $30 \mathrm{keV}$ \\
\hline Vacío de cámara & 3 - $150 \mathrm{~Pa}$ \\
\hline Campo de visión & $7.7 \mathrm{~mm}$ en WDanalytical $10 \mathrm{~mm}$ \\
\hline Energía de haz de electrones & $200 \mathrm{eV}$ a $30 \mathrm{keV}$ \\
\hline Sonda actual & $1 \mathrm{pA}$ a $2 \mu \mathrm{A}$ \\
\hline Velocidad de escaneo & De $20 \mathrm{~ns}$ a $10 \mathrm{~ms}$ por píxel \\
\hline Tamaño de la imagen & $16,384 \times 16,384$ píxeles \\
\hline
\end{tabular}


Figura 2. Microscopio electrónico Tescan-Vega LMU

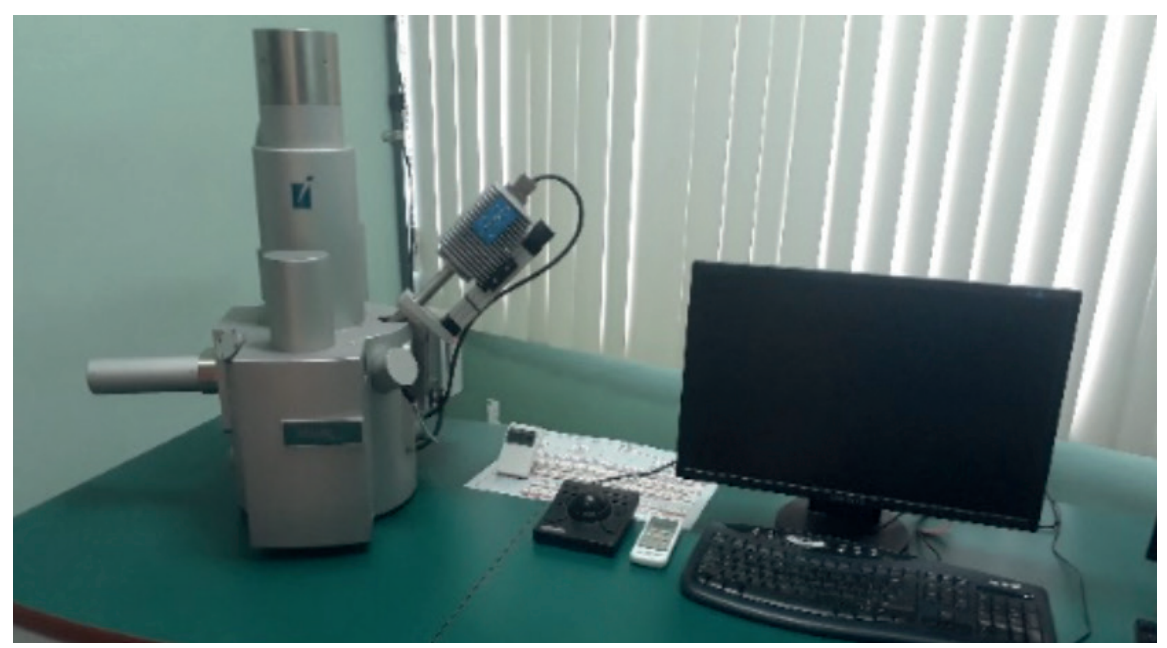

\section{Resultados y discusión}

En este apartado se presentan y discuten los resultados obtenidos al aplicar los dos métodos de ensayos (estereoscopía y la microscopía electrónica de barrido)

\subsection{Estereoscopía de los microfiltros}

En el análisis de estereoscopía se presentan las imágenes obtenidas en las mallas de los microfiltros de los inyectores de los vehículos Aveo y Corsa. En la Figura 3 se observa la malla de uno de los microfiltros del vehículo Aveo luego de recorrer 3000 km con gasolina 87 octanos. Los cuatro microfiltros de los inyectores presentaron una tendencia de obstrucción del 4 \% en la superficie de la malla.

Figura 3. Malla de microfiltro del inyector Aveo (zoom: 1.25x, 5x, combustible 87 \% octano)

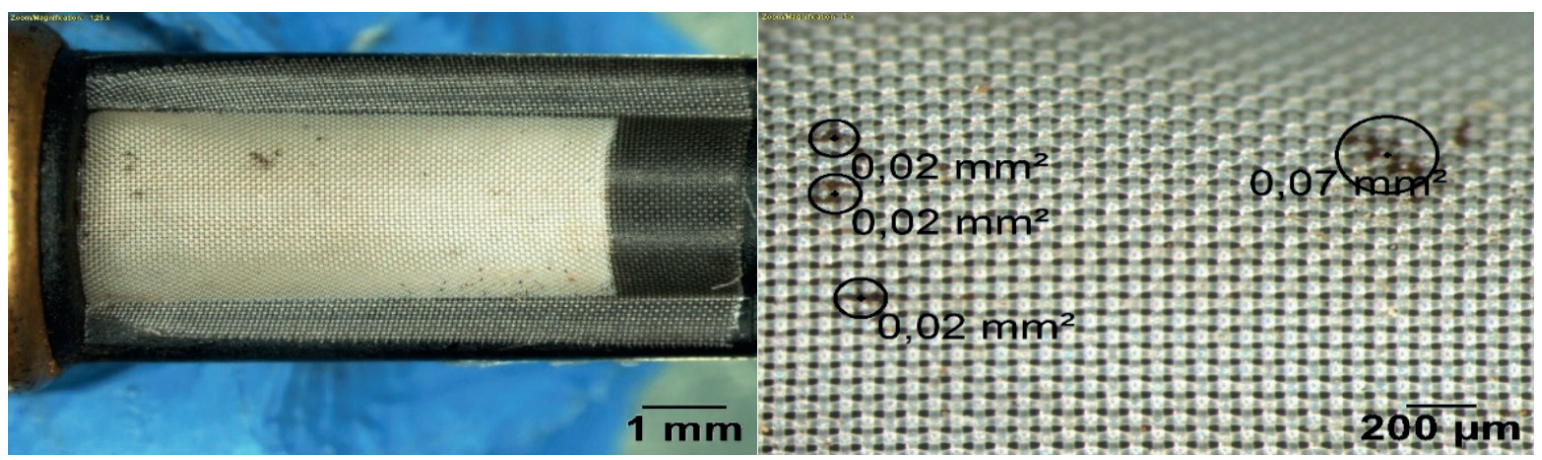

En la Figura 4 se observa la malla de uno de los microfiltros del vehículo Corsa luego de recorrer $3000 \mathrm{~km}$ con gasolina al $87 \%$ octano. Los cuatro microfiltros de los inyectores presentaron una tendencia de obstrucción del 9 \% en la superficie de la malla. 
Figura 4. Malla de microfiltro del inyector Corsa (zoom: 1.25x, 5x, combustible 87 \% octano)

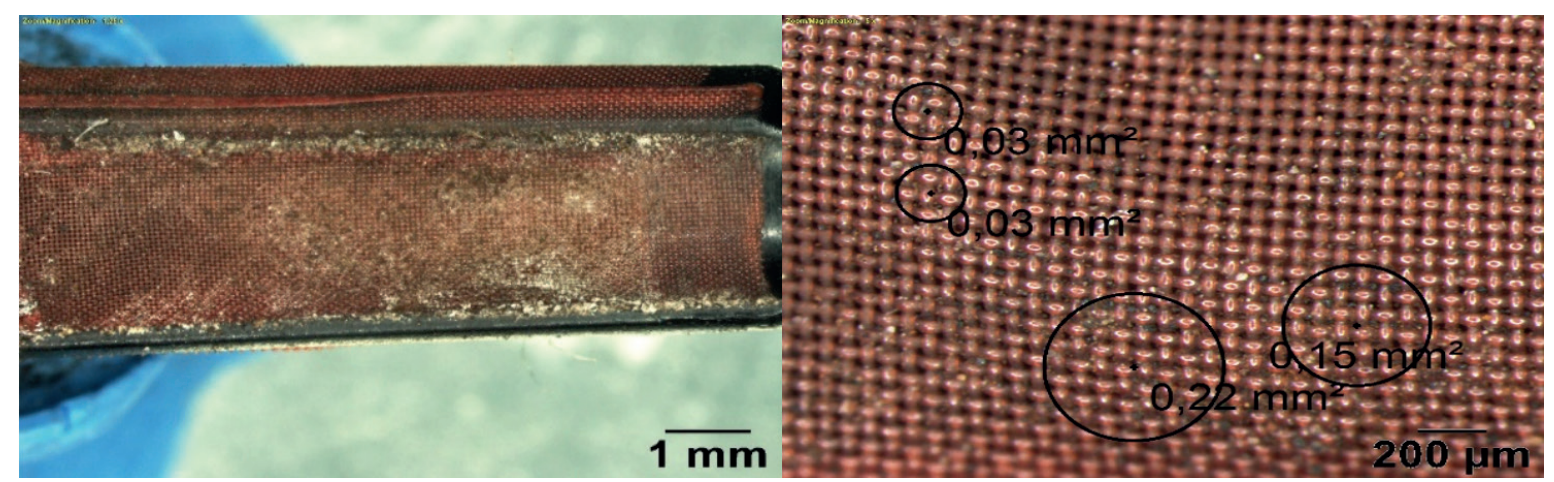

En la Figura 5 se observa la malla de uno de los microfiltros del vehículo Aveo luego de recorrer 3000 km con gasolina con un 5 \% de etanol anhidro. Los cuatro microfiltros de los inyectores presentaron una tendencia de obstrucción de menos del 1 \% en la superficie de la malla.

Figura 5. Malla de microfiltro del inyector aveo, (zoom: 1.25x, 5x, combustible al 5 \% etanol)
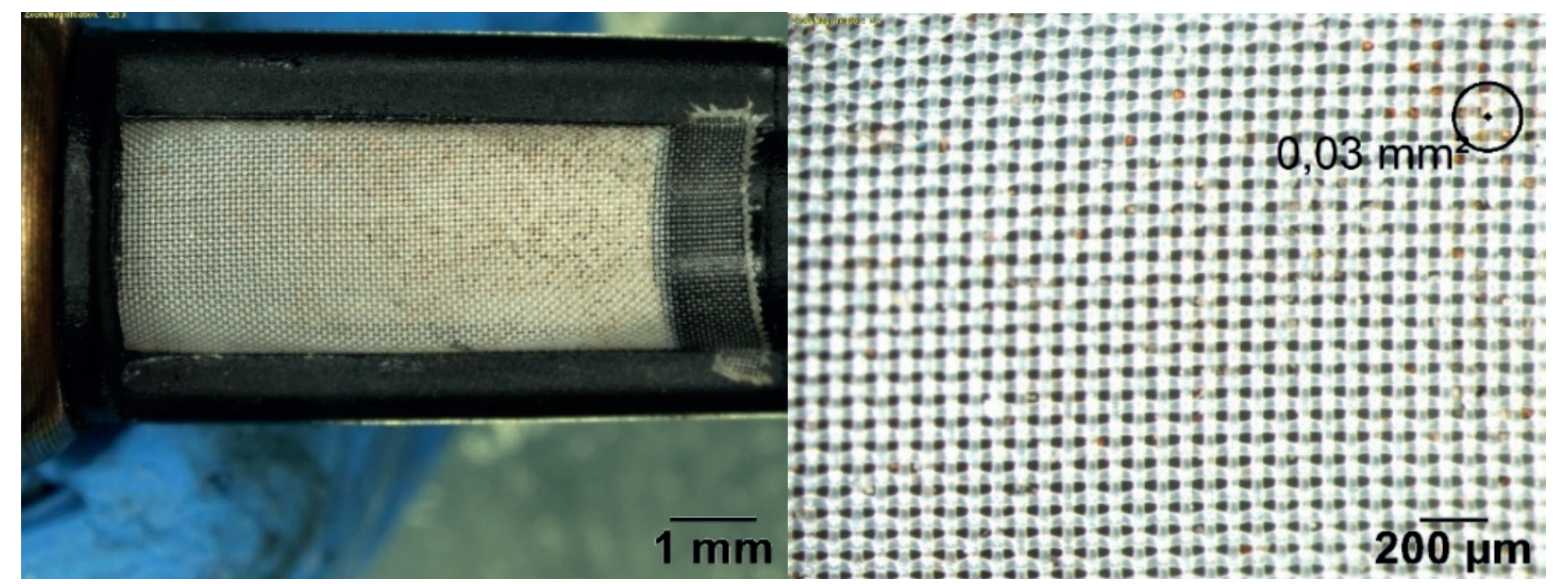

En la Figura 6 se observa la malla de uno de los microfiltros del vehículo Corsa luego de recorrer 3000 km con gasolina y 5 \% de etanol anhidro. Los cuatro microfiltros de los inyectores presentaron una tendencia de obstrucción de menos del 1 \% en la superficie de la malla.

Figura 6. Malla de microfiltro del inyector corsa (zoom: 1.25x, 5x, combustible al 5 \% etanol)

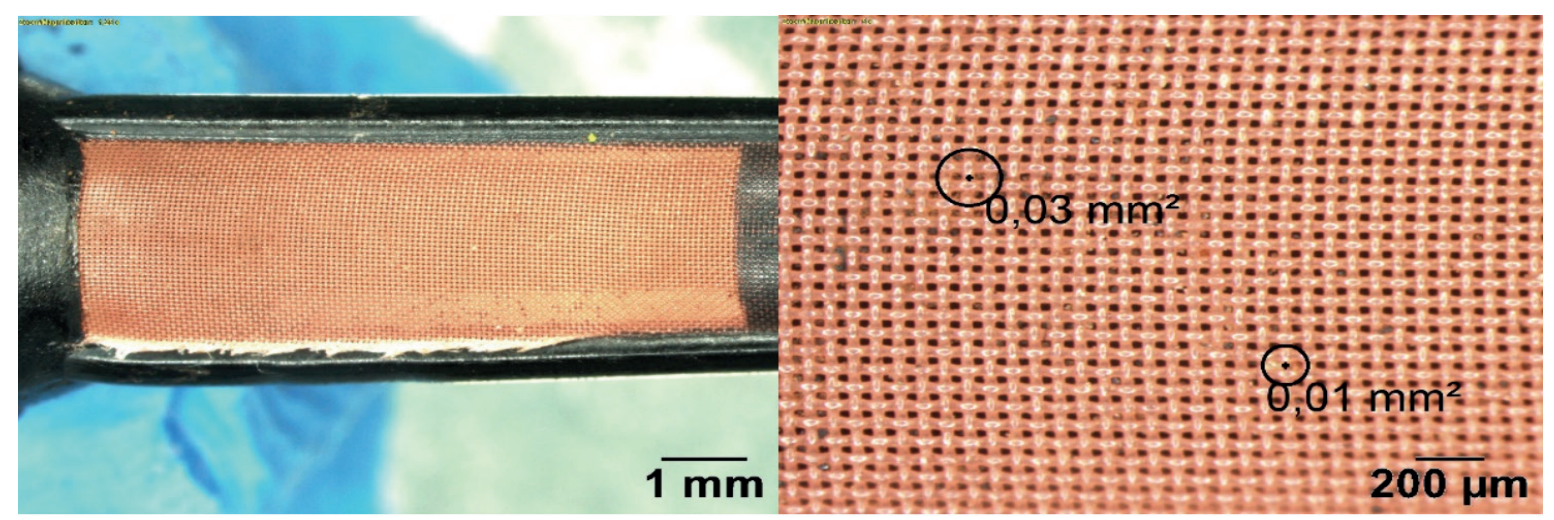


En el caso de los dos vehículos se presenta una reducción en el área de obstrucción de la malla de los microfiltros de los inyectores al usar gasolina de 87 octanos con $5 \%$ de etanol anhidro, siendo la diferencia más notoria en el caso del vehículo Corsa; esto se debe a que el etanol es un buen disolvente y purificador, resultado que coincide con los referenciados por Cornejo et al. (2017); también provoca que los residuos de combustible que obstruyen la malla de los microfiltros de los inyectores se diluyan. Esta comparación se puede realizar gracias a una vista tridimensional de objetos percibidos mediante visión binocular, según método referido por Cárdenas, Morales, y Ussa (2015).

\subsection{Microscopia Electrónica de Barrido de los inyectores}

En la Figura 7 se representa la imagen tomada a los residuos de los microfiltros de los inyectores mediante la MEB al emplear gasolina de 87 octanos, visibilizándose la presencia de materiales como zinc, hierro, azufre, silicio, calcio y fósforo resultados de la operación de 3000 km de recorrido por el vehículo.

Figura 7. Residuos en los filtros de inyector de la gasolina extra, 150x

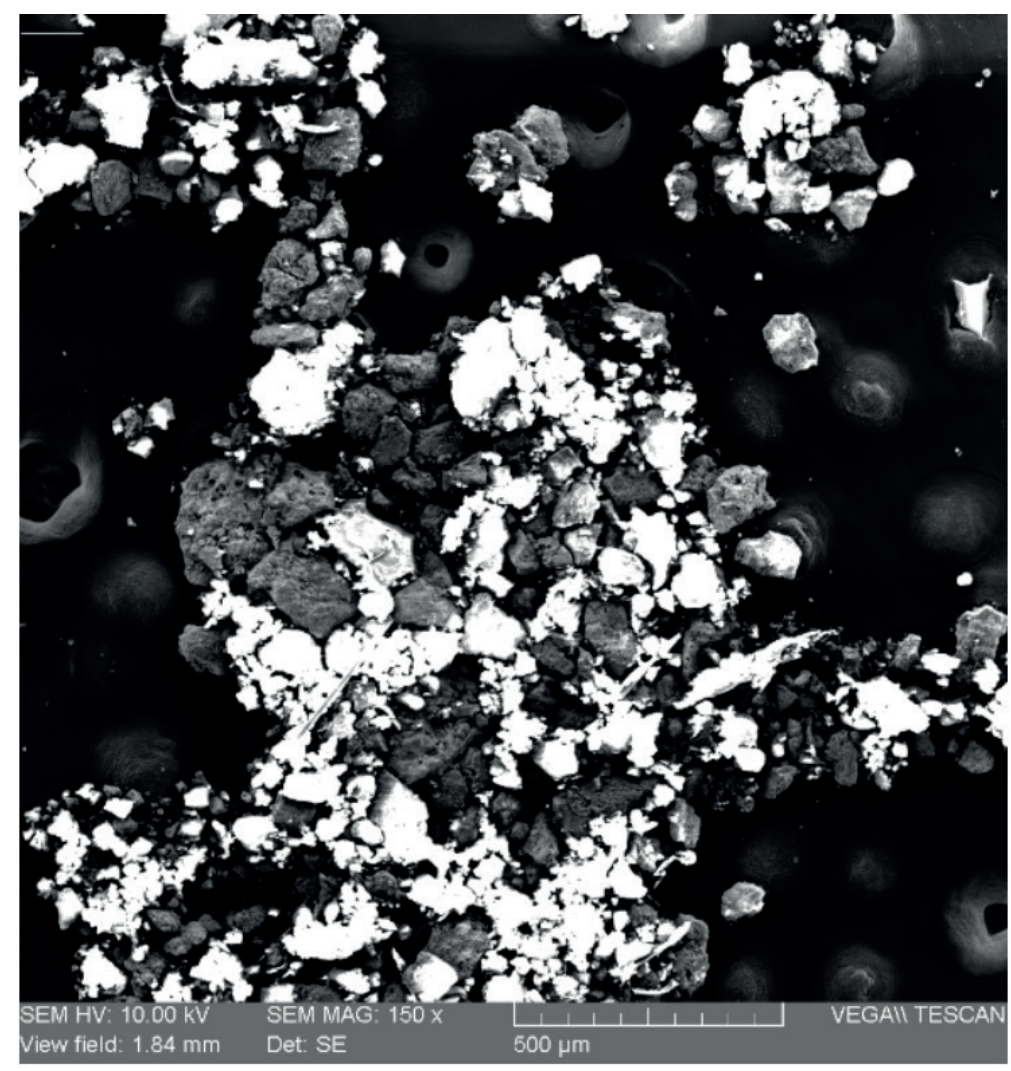

En la Figura 8 se observa la muestra en el análisis MEB en residuos de la mezcla de gasolina con $5 \%$ de etanol anhidro obtenidos en los microfiltros de los inyectores. Se identifican elementos que se han acumulado como cobre, hierro, azufre, silicio, y calcio resultados del trabajo del vehículo en el período de prueba. 
Figura 8. Residuos de gasolina al $5 \%$ etanol, 150x

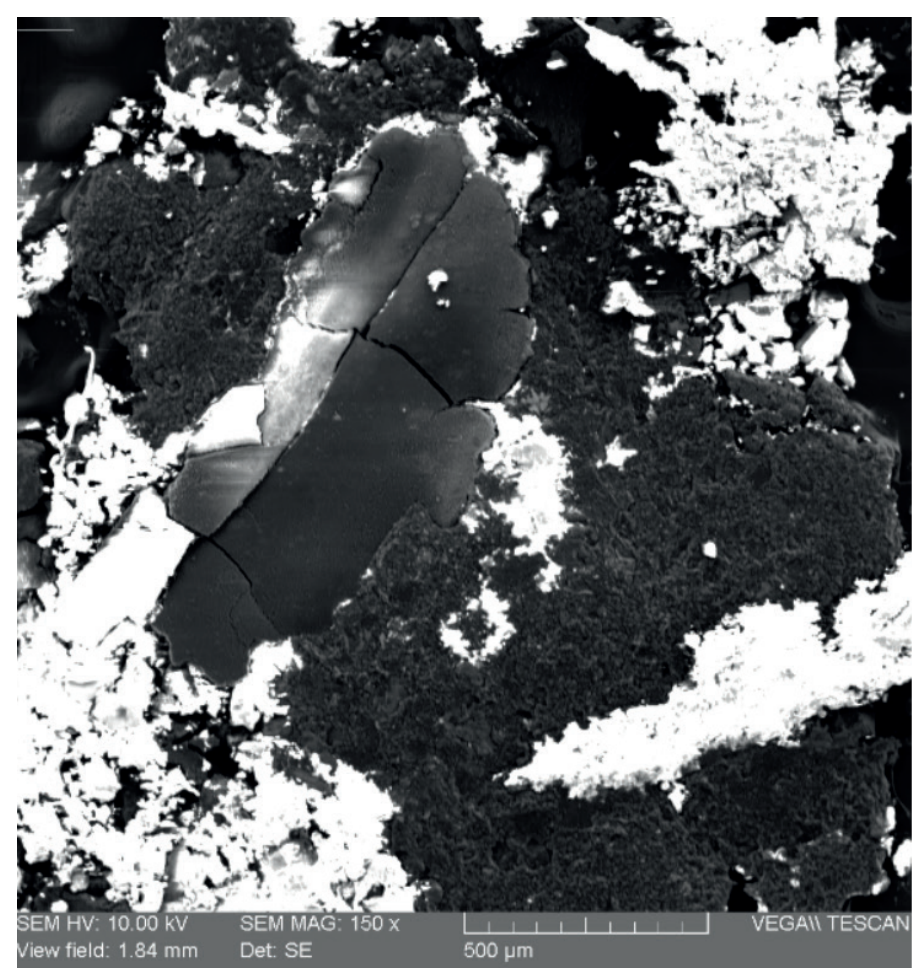

En la Tabla 5 se presenta un valor alto de cobre (alrededor del 14\%) en la gasolina de 87 octanos al 5 \% etanol, residuos de este material por partículas del sistema de combustible y del propio combustible. También se evidencia silicio, donde se muestra que es parte de agentes externos provocados por el repostaje o el abastecimiento y manejo del combustible. Se resalta en la Tabla 5 mayor presencia del elemento azufre (con el 4 \%) en los residuos de gasolina de 87 octanos con 5 \% de etanol, a diferencia del $1 \%$ en los residuos de la gasolina de 87 octanos; la mayor presencia de azufre provoca que durante la combustión se generen mayores emisiones de dióxido de azufre $\left(\mathrm{SO}_{2}\right)$, el cual es liberado a la atmósfera donde tiene efectos tóxicos (Gary y Handwerk, 2001; Celi-Ortega, Llanes-Cedeño, Rocha-Hoyos, Leguísamo-Milla, Peralta-Zurita, y Salazar, 2018) y genera un gran deterioro al ambiente. El potencial para reducir las emisiones de gases de efecto invernadero es un beneficio sustancial de los combustibles de bajo azufre (Alcántar y Cruz, 2011), para el caso objeto de estudio la gasolina de 87 octanos.

Tabla 5. Elementos de las muestras de gasolina de 87 octanos y gasolina al 5 \% etanol

\begin{tabular}{|l|c|c|}
\hline \multicolumn{1}{|c|}{ Elementos } & Gasolina de 87 octanos & $\begin{array}{c}\text { Gasolina de 87 octanos } \\
\text { al 5 \% etanol }\end{array}$ \\
\hline Zinc (Zn) & $3 \%$ & $11 \%$ \\
\hline Hierro (Fe) & $1 \%$ & $4 \%$ \\
\hline Azufre (S) & $1 \%$ & $4 \%$ \\
\hline Silicio (Si) & $3 \%$ & $1 \%$ \\
\hline Calcio (Ca) & $7 \%$ & \\
\hline Fósforo (P) & $1 \%$ & $14 \%$ \\
\hline Cobre $(\mathrm{Cu})$ & & \\
\hline
\end{tabular}




\section{Conclusiones y recomendaciones}

El análisis de estereoscopía en los dos vehículos evidenció la reducción en la obstrucción de los filtros al usar gasolina de 87 octanos al 5 \% de etanol anhidro en comparación con la gasolina al $87 \%$.

Con la MEB se evidencia que existe mayor presencia de azufre, con un $4 \%$ en los residuos al emplear gasolina con $5 \%$ de etanol, a diferencia del $1 \%$ en los residuos al utilizar gasolina de 87 octanos.

Si bien se obtienen menos obstrucciones en los microfiltros al emplear gasolina al $5 \%$ de etanol, también se obtiene un aumento de azufre en los depósitos, lo cual provocaría mayores emanaciones de dióxido de azufre $\left(\mathrm{SO}_{2}\right)$ al ambiente con consecuencias negativas a la salud.

\section{Bibliografía}

Alcántar, F. S., y Cruz, J. (2011). Análisis de la distribución de azufre en productos, emisiones de SOx y la recuperación del mismo en el sistema nacional de refinación. Revista internacional de contaminación ambiental 27(2), 153-163.

Aradi, A. A., Evans, J., Miller, K., y Hotchkiss, A. (2003). Direct injection gasoline (DIG) injector deposit control with additives (2003-01-2024). SAE Technical Paper.

Arters, D. C., y Macduff, M. J. (2000). The effect on vehicle performance of injector deposits in a direct injection gasoline engine (No. 2000-01-2021). SAE Technical Paper.

Andrade, J. (2018). Caracterización del comportamiento del inyector de un sistema de inyección electrónica indirecta multipunto de un MEP para combustibles no tradicionales (Master tesis, UISEK, Quito).

Cárdenas Quiroga, E. A., Morales Martín, L. Y., y Ussa Caycedo, A. (2015). La estereoscopía, métodos y aplicaciones en diferentes áreas del conocimiento. Revista Científica General José María Córdova, 201-219.

Cedeño, E. A. L., Carguachi-Caizatoa, J. B., y Rocha-Hoyos, J. C. (2018). Evaluación energética y exergética en un motor de combustión interna ciclo Otto de 1.6 L. Enfoque UTE, 9(4), 221-232.

Cedeño, E. A. L., Rocha-Hoyos, J. C., Zurita, D. B. P., y Milla, J. C. L. (2018). Evaluación de emisiones de gases en un vehículo liviano a gasolina en condiciones de altura. Caso de estudio Quito, Ecuador. Enfoque UTE, 9(2), 149-158.

Celi-Ortega, S., Llanes-Cedeño, E., Rocha-Hoyos, J., Leguísamo-Milla, J., Peralta-Zurita, D., y Salazar, P. (2018). Comportamiento de las emisiones en motor de encendido provocado a base de etanol a $2850 \mathrm{msnm}$. UTCIENCIA, 5(1), 1-10.

CINAE. (2018). Anuario De La Industria Automotriz Ecuatoriana. Quito: David Molina.

Cornejo, A., Barrio, I., Campoy, M., Lázaro, J., y Navarrete, B. (2017). Oxygenated fuel additives from glycerol valorization. Main production pathways and effects on fuel properties and engine performance: A critical review. Renewable and Sustainable Energy Reviews, 79, 1400-1413. https://doi. org/10.1016/j.rser.2017.04.005

El-Faroug, M. O., Yan, F., Luo, M., y Fiifi Turkson, R. (2016). Spark ignition engine combustion, performance and emission products from hydrous ethanol and its blends with gasoline. Energies, 9(12), 984.

Gary, J., y Handwerk, G. (2001). Petroleum Refining Technology and Economics. New York: Editorial Marcel Dekker Inc - Cuarta Edición.

Guardia, Y., J. Márquez, V. Sánchez, E. A. Llanes-Cedeño, J.C. Rocha-Hoyos y D.B. Peralta. (2018). Mejoras a la asignatura Diseño Estadístico de Experimentos para estudiantes de la carrera de Ingeniería Mecánica. Revista ESPACIOS, 39(30).

Lindgren, R., Skogsberg, M., Sandquist, H., y Denbratt, I. (2003). The influence of injector deposits on mixture formation in a DISC SI engine (No. 2003-01-1771). SAE Technical Paper. 
Llanes-Cedeño, Rocha-Hoyos, Peralta, Martínez, y Celi. (2018). Project-based learning case of study education in automotive mechanical engineering. Revista ESPACIOS, 39(25).

Merchán, M. V. N., Moromenacho, E. G. P., Milla, J. C. L., y Cedeño, E. A. L. (2018). Identificación Del Modo De Trabajo De Un Motor GDI Al Variar La Altura Mediante Una Prueba Estática a Ralentí y 2500 RPM. INNOVA Research Journal, 3(7), 61-70.

Páez, C. (2008). Gestión de la Calidad del Aire. Carlos Páez Pérez®, Quito, Ecuador, 2-6.

Pérez Salinas, C. F., Moya, E., y Coello, D. (2018). Uso de un arreglo ortogonal para el análisis del proceso de electroerosión por penetración con electrodos de forma de grafito y cobre sobre micro-fundición de aluminio. Enfoque UTE, 9(3), 67-79.

Proaño Sánchez, J. E. (2018). Análisis Forense de un Cigüeñal Mediante Técnica de Fractografía y Simulación Mediante Elementos Finitos (MEF) (Bachelor's thesis, Quito).

Rocha-Hoyos, Juan C., Tipanluisa, Luis E., Zambrano, Víctor D., y Portilla, Ángel A. (2018). Estudio de un Motor a Gasolina en Condiciones de Altura con Mezclas de Aditivo Orgánico en el Combustible. Información tecnológica, 29(5), 325-334. https://doi: 10.4067/S0718-07642018000500325

Secretaria de Ambiente. (2016). Actualización del Plan de Manejo de la Calidad del Aire 2005 - 2010. Recuperado de http://www.quitoambiente.gob.ec/ambiente/index.php/ bibliotecadigital/ category/61-politicas-yplaneacion ambiental?download=529:calidad del-aire-final.

Song, H., Xiao, J., Chen, Y., y Huang, Z. (2016). The effects of deposits on spray behaviors of a gasoline direct injector. Fuel, 180, 506-513. https://doi.org/10.1016/j.fuel.2016.04.067

TESCAN (2018) Microscopía Electrónica de Barrido. Recuperado de https://www.tescan.com/en-us/technology/sem

Vega, W.H., E.A. Llanes-Cedeño, J. V. Molina y J. C. Rocha-Hoyos (2018). Revisión de las Características de Modelado y Optimización para el Diseño del Sistema de Suspensión Macpherson, Inf. Tecnol., 29(6), 221-233.

Villavicencio Cevallos, J. G. (2016). Obtención de alúmina activada y su aplicación como soporte de catalizador (Bachelor's thesis, Quito, 2016).

Wang, B., Jiang, Y., Hutchins, P., Badawy, T., Xu, H., Zhang, X., y Tafforeau, P. (2017). Numerical analysis of deposit effect on nozzle flow and spray characteristics of GDI injectors. Applied Energy, 204, 1215-1224.

Zhao, F., Lai, M. C., y Harrington, D. L. (1999). Automotive spark-ignited direct-injection gasoline engines. Progress in energy and combustion science, 25(5), 437-562. 